\title{
Mobile physical activity planning and tracking: a brief overview of current options and desiderata for future solutions
}

\author{
Maged N. Kamel Boulos ${ }^{1}$, Stephen P. Yang ${ }^{2}$ \\ ${ }^{1}$ School of Information Management, Sun Yat-sen University, Guangzhou 510006, China; ${ }^{2}$ State University of New York College at Cortland, \\ Cortland, NY, USA \\ Contributions: (I) Conception and design: MN Kamel Boulos; (II) Administrative support: MN Kamel Boulos; (III) Provision of study materials or \\ patients: All authors; (IV) Collection and assembly of data: All authors; (V) Data analysis and interpretation: MN Kamel Boulos; (VI) Manuscript \\ writing: All authors; (VII) Final approval of manuscript: All authors. \\ Correspondence to: Maged N. Kamel Boulos. School of Information Management, Sun Yat-sen University, Guangzhou 510006, China. \\ Email: mnkboulos@mail.sysu.edu.cn; mnkboulos@ieee.org.
}

\begin{abstract}
Consistent and enjoyable physical activity (PA) can be a crucial component to improving or maintaining one's overall health status. Using advanced features on smartphones (GPS, Bluetooth, motion sensing, etc.) coupled with an app or game that is able to assist mobile users to not only track location, but also to interact socially with others based in real-life (IRL), virtual reality (VR), or alternate-reality (ARG), has the potential to give health experts better tools to encourage higher compliance to protocols, rehabilitation, behaviour change and health outcomes. This paper outlines the available mHealth apps that capitalize on pervasive smartphone features coupled with sensors, and suggests which features might impact future PA patterns. The authors argue that the ultimate mobile PA planning and tracking app/platform will be the one capable of supporting both precision and accuracy health (offering truly individualized PA advice and coaching while preserving user privacy) and precision and accuracy public health (providing public health decision makers with community-level PA indicators obtained from app data aggregates of user populations).
\end{abstract}

Keywords: Physical activity; mHealth; digital health; digital public health

Received: 02 September 2019; Accepted: 12 December 2019; Published: 20 January 2021.

doi: $10.21037 /$ mhealth.2020.01.01

View this article at: http://dx.doi.org/10.21037/mhealth.2020.01.01

\section{Introduction}

Physical activity (PA) is critical for achieving and maintaining good health and for the prevention and control of a widerange of chronic diseases, including type 2 diabetes (and its many complications), heart disease, joint disease, and certain types of cancer and dementia, but is not as good for calorie burning or for offsetting a bad, sugar and calorieladen diet despite the claims promoted by 'big food' companies $(1,2)$. Our bodies are very energy-efficient machines! It takes at least half hour of brisk walking to just burn two McVitie's digestive biscuits! [Vox has a 2017 report intended for non-clinical lay people explaining this further and drawing on research evidence from dozens of studies (3).] The inherently flawed "calories in/calories out" CICO model of energy balance should be tempered with sensible food choices and satiety. Unlearning poor eating habits and avoiding ultra-processed foods (with high amounts of refined carbohydrates) are a healthier, more effective and sustainable long-term strategy than just attempting to burn a couple hundred more calories through exercise. Some individuals have a small but significant rise in risk of developing diabetes with each 150-calorie increase in sugar availability per day (4).

Individual PA and its health gains are dependent on a number of location-specific factors, including the availability, positioning and accessibility of PA POIs (points of interest), such as local green spaces/parks and covered sports spaces and gym facilities (especially important during temperature extremes and heavy rain/snow seasons). 
Additional neighbourhood walkability indicators that affect PA levels and weight gains of residents include pollution (air, light and sound/noise), crowdedness, crime rates, traffic/ road network safety, and even individual age, gender and genetic predisposition differences (5-7).

Despite the ubiquity of the 10,000-daily-step advice to be healthy, there is very little medical evidence to back up this assertion (8). In fact, a more moderate figure of between 6,000 and 7,000 steps/day or 150 minutes of moderate exercise/week is recommended. [See, for example, the UK Guidance (9).] Among older women, a recent study found that as few as 4,400 steps per day was significantly related to lower mortality rates compared to 2,700 steps per day (10).

However, as we will be discussing below, the accuracy of current mobile PA tracking solutions is questionable when determining the exact number of steps, a person has undertaken during a given day!

Two modes of training that have become popular are high intensity interval training (HIIT) and CrossFit. They both offer shorter overall workout times with a variety of different exercises typically timed with very little rest between exercises and sets. This contrasts with longer traditional workouts that can be repetitive and more rigid. Although there are advantages to both types of workouts, HIIT seems to fit our modern lifestyles of instant gratification and lots of variety.

\section{Current mobile applications for PA planning and tracking}

\section{App categories}

These can be divided into five different categories. Apps with GPS (Global Positioning System) logging seem to be popular in mobile marketplaces, likely due to the popularity of both running and cycling. However, these kinds of apps do not yet support universal training (e.g., at home, in the office, outdoors, etc.) and are lacking in terms of activity recognition and segmentation (see later below) as well as contextually-suited exercises.

\section{GPS trackers}

Examples under this category include Arion (11), MayMyRun by Under Armour (12), Nike + Run Club (13), RunKeeper (14), Strava (15), or Runtastic (16) and their former miCoach app, Sports Tracker (17). After the activity is complete, the GPS traces can be reviewed and viewed with respect to heart rate, step counts, elevation, speed, or
RPM (revolution per minute - bike). Further information from the phone's built-in sensors, such as accelerometer or magnetometer data, is not usually included; however, most applications integrate Bluetooth/ANT+ (18) heart rate monitors and/or the Apple Watch heart-rate sensors, while some also combine motion sensors (wearable technology) integrated into shoes, socks, sporting equipment [Sensoria(19)] or online music platforms such as Spotify [Map My Run, Rock My Run (20), and Runkeeper].

\section{Workout and route planners}

These applications, such as Fiit (21), Fitbod (22), JEFit (23), and Johnson \& Johnson's 7 Minute Workout (24), accompany goal-directed workout such as bodybuilding or weight training. They typically contain exercises organised by body parts or muscle groups, and log exercise performances (quantitative, not qualitative) while integrating algorithms to vary workouts and avoid monotony. While the functionality and usability of apps in this category greatly varies, very few of them offer personalized exercise monitoring in terms of quality or individual workouts. Some apps have capitalized on the popularity of HIIT, which provides a series of exercises usually with a short timer function, and opposing sets of exercises (e.g., upper body $v$ s. lower body, push $v s$. pull, or large muscle group $v s$. small muscle group). Similar to the early Tabata-based timer apps (Tabata is a HIIT programme first proposed by Japanese scientist Dr. Izumi Tabata at the National Institute of Fitness and Sports in Tokyo), many of these apps are popular as they provide varying types of exercises in a short time span. Many apps, such as Class Pass Fiit (21), Daily Burn (25), Peloton (26) and WellBeats (27), provide access to on-demand fitness classes or trainers, some of which occur in real-time, and companies like Peloton use their integrated cycles and treadmills. Another popular cycling app that integrates at home is Zwift (28) which received $\$ 166$ million in funding for its growing online community of cyclists, cumulating into an eSports world championships (29) (Figure 1). In 2018, the Seminar on eSports, Exergaming, and Fantasy Leagues was held in Finland where one researcher (32) posited "watching other people play becomes meaningful "beyond play"'. And perhaps watching athletes compete in real-time on a virtual course might be as popular as the recent Fortnite solo competition, where the winner, a teenager, won almost $\$ 3$ million (33). Ironically, or perhaps not, the event was held in a tennis stadium [watch event video at (34)]. Is this a case of art imitating life, or life imitating virtual? 


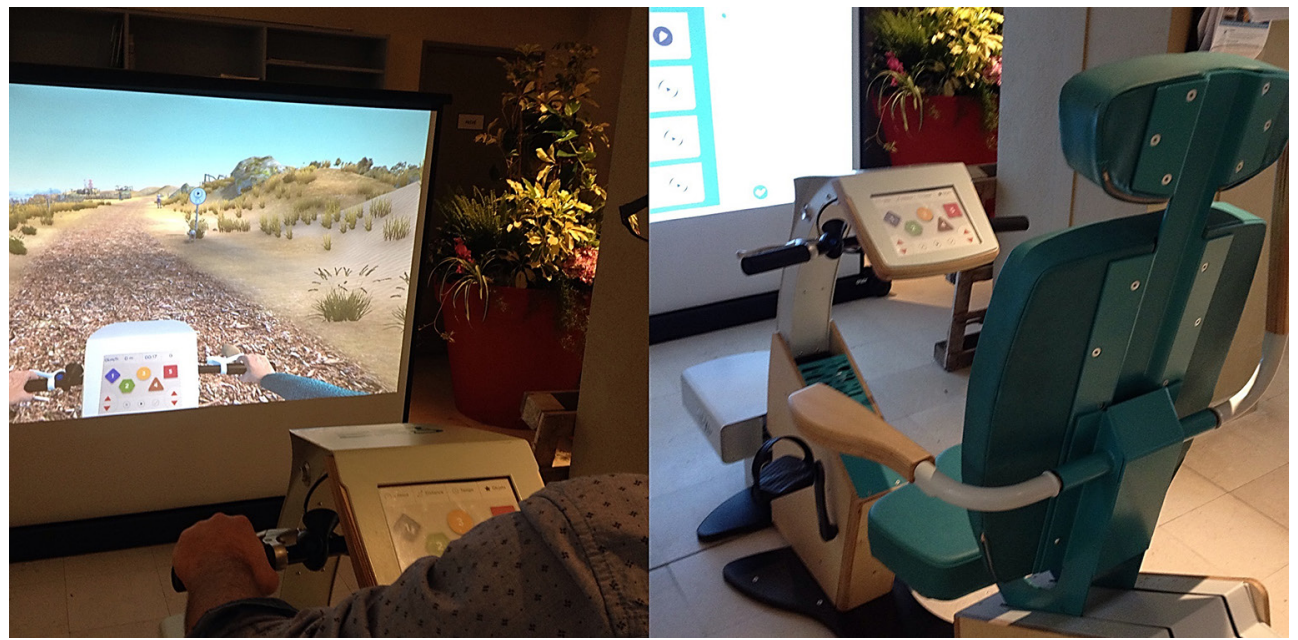

Figure 1 Cybercycling using Cycléo from Cottos Medical (30). Photos by Maged N. Kamel Boulos, October 2017, Angers, France (31). Claimed health benefits include improvement of cognitive functions in older people. Cycléo is a good example of a digital physical activity tracking application with virtual reality and gaming elements, although not offered in a truly mobile form factor.

Despite being a limiting factor for most apps, providing dynamic and progressively more challenging workouts is a new feature for Fitbod (22), which uses advanced algorithms to program user's workout specific to her/his strengthtraining goals, and integrates to the Apple Watch to track progress instantly. The feedback that users input after each set is supposed to provide an indication to the algorithm to ease or intensify the workout based on user's goals. What is not known is if the heart rate monitor data are also included in the algorithm to determine PA exertion levels.

Exercising outdoors is not always simple to do especially when there are environmental triggers/allergens, if someone suffers from seasonal allergies, asthma, or there is a pollution problem. Several apps have been developed to assist users in planning routes based on latest updates from air quality monitoring stations typically located in cities, including Air (35), Breezometer (36) and Klarify (37). Each offering provides users with access to real-time data on air quality and weather forecasting, while providing alternate route planning on necessary travel days. Breezometer is unique in that it exposes an API [Application Programming Interface (38)] that will allow other apps to integrate its air quality data, thus providing a more robust picture for exercise or event planning. For example, someone with asthma walking to work could plan their route daily to avoid heavy pollution or pollen areas, or at specific times of the day with the least triggers/likelihood of having an asthmatic event. Teachers could also decide which park to bring their students to based on the air quality forecast for the next day, especially if they know some of their students have asthma.

Another form of pollution that affects user's PA levels is noise pollution, and with the newest Apple Watch and software update, accurate sound decibel monitoring is now an active feature (39). Like the air pollution monitoring via apps, noise monitoring via a collective of Apple Watch users might one day provide less noisy travel routes for those wanting a peaceful commute or walk.

\section{Exercise books}

These applications, as seen, for example, in a number of Pilates apps on offer on the Play Store (40), provide a browsable compendium of exercises, with few if any smartphone sensors activated, compared to the other genres, but the deepest background information on correct exercise performance and health-related issues. Sworkit Fitness by Nexercise (41) has 17 million downloads and is a popular choice for those looking for a guided workout. More and more of these stand-alone apps are integrating adaptive workouts using algorithms and/or more individualized programming including to upgrade to a personal coaching session.

\section{Daily living PA monitoring}

These applications allow to constantly track the paths taken during the day, recording, for example, the time spent walking, cycling and running by using both the GPS and the inertial sensors of the smartphone. Moreover, they usually (rather crudely) estimate the calories burned while 
performing each activity of the day. They usually feature a step counter and allow setting simple goals of daily activity in order to enable a healthier life style. One of the most well-known of these apps is Google Fit (42), which earned the 'blessings' of the World Health Organisation (WHO) and the American Heart Association (AHA). The Google Fit platform works with dozens of third-party apps (43). Another example is the WeRun applet within WeChat (44). Daily living PA monitoring is particularly important for reinforcing the habit of having appropriate PA levels in the daily routine or when following a prescribed exercise plan $(45,46)$.

A current app that harnesses daily living PA monitoring (with phone or fitness tracker) is Sweatcoin (47). The more steps a user takes the more 'sweatcoins', a kind of digital currency, s/he can earn. One feature that stands out with the rewards structure is the philanthropic possibility, in that users can donate their 'sweatcoins' to charities. As of mid-November 2019, their website states that more than 6 billion (ten to the twelfth power) steps have been converted to date, and one of their goals is to make sweatcoins universally accepted as a form of payment.

Besides daily living PA monitoring, there is an ongoing trend led by Apple and other manufacturers to develop gadgets and apps that continuously monitor other aspects of a user's health and take appropriate actions accordingly. While some Apple Watch features are quite utilitarian, including alerting police of being stranded on a paddle board (48) and monitoring heart rate during exercise sessions, it can also be highly accurate as an electrocardiogram (ECG) to determine cardiac events/arrythmias and alert medical professionals (49). Recently a woman with epilepsy was located because of her Apple Watch fall detection feature alerted her caregiver (50). Given the plethora of features and health tracking possibilities, some envision the Apple Watch as the "control centre for your health" (51). It can track daily PA, exercise, medications, cardiac rehabilitation and monitoring, pulse oximetry, etc., and seamlessly integrates with the Apple Health app, which continues to be used by researchers conducting clinical trials as the data being transmitted and stored conforms to HIPPA standards (US Health Insurance Portability and Accountability Act of 1996 covering data privacy and security provisions for medical information). Several studies have already been conducted using Apple Health ResearchKit (52), including research about patients with autism, Parkinson's disease, epilepsy, and concussions.

Some health insurers, including AETNA (53) are also hoping fitness tracker might be a solution for lower healthcare costs and better health outcomes. In the United Arab Emirates (UAE), fitness trackers were given out in hopes of getting their country's population more active by setting a goal of collecting 1 billion steps before the 2019 Special Olympics World Summer Games (54).

\section{Game-based apps}

Although a good number of apps in the four main categories above involve a gamification feature of some sort, one can also consider a distinct category dedicated to apps that are primarily PA games (digital exergames) $(55,56)$. Exergames require the game player to physically move in order to progress in the game and accomplish specific goals. Some games capture PA as steps and converts it into points; for example Wokamon (57) is a pedometerbased app that converts steps to help a player-adopted ingame monster (virtual pet) grow and allows players to collect more 'wokamons' for that purpose as they move, walk and run. Another genre of exergames are audio-based, and the most popular of these is Zombies, Run! (58), with over one million downloads. Using narrated stories and sounds of zombies chasing the player, as s/he increases her/ his real-life movement, the sounds from chasing monsters fade away. In essence, players can stay alive in the game by moving more in the real world, which might be a good metaphor in general for a healthier lifestyle. Another audiobased exergame is The Walk (59), which was created with the NHS (England's National Health Service) and UK's Department of Health. The game plot is designed to encourage PA, as the game map is the players' real world. To save the world after a bomb explodes in a London subway station, players need to walk the length of the UK.

Many games use GPS, cameras and motion sensors to provide an augmented reality game (ARG). Pokémon GO is the most well-known ARG and has over one billion downloads. It dominates the location-based game genre with $84 \%$ of users (60). Based originally on the Ingress (61) platform, Pokémon GO has now been re-skinned into the Harry Potter wizarding universe (62).

\section{Wearable sensors}

Applications relying exclusively on the smartphone and its built-in sensors have been proposed in the literature (and some have been marketed to consumers); however, in a good number of these applications, the smartphone has to be strictly worn on the waist to ensure accurate 
measurements (63) or, in other studies, on the waist or in the pocket (64). Extensive research work is necessary in order to make these applications reliable without restrictions regarding the position of the phone. There also very wide variations in the range and quality/accuracy of the sensors that are built in various smartphone models from same and various manufacturers, so the same app (software) might yield different levels of accuracy when run on different handsets.

The introduction of specialized wearable sensors that communicate with the smartphone has been a recent trend that proved more popular with consumers. These sensors are relatively low-cost and have reduced dimensions, being available in the shape of pendants, bracelets/wristbands or smartwatches, which is an advantage when practicing more intense sports. Currently, several devices equipped with inertial sensors are being used and commercialized for activity monitoring, relying (mainly) on a step counter (pedometer), and providing estimates of the travelled distance and energy expended. (The latter tends to overemphasise the flawed and over simplistic model of calories in (diet)/calories out (exercise) energy balance/imbalance.) Some examples are the offerings from FitBit ${ }^{\circledR}(65)$, the once popular Nike+ FuelBand (66), Omron ${ }^{\circledR}$ activity monitoring products (67), and Apple Watch (68), among others.

As of 2019, stand-alone fitness apps are seeing their market encroached on by well-established hardware (healthoriented smartwatches, etc.) makers and their ecosystems (Apple, Asus, Withings and others). By controlling the hardware, Apple, for example, is also controlling the software and user data. But not all hardware makers are following in Apple's footsteps. Unlike Apple, Google has shown little interest in adopting a similar approach with a smartwatch of its own, but this position is likely to change at some future point with Google's acquisition of Fitbit. [For a detailed treatment of these subjects, the reader is referred to the following news media analyses: (69-71).]

\section{Limitations and caveats in current mobile app and wearable solutions}

\section{Accuracy concerns}

When it comes to PA tracking apps on smartphones or dedicated wearables/bands (steps/distance, etc.), there is a growing body of research evidence and other reports in the grey literature about the accuracy/inaccuracies of their measurements, which can vary by as much as $50 \%$ or more between different models within the same brand/ manufacturer and across different brands/manufacturers, e.g., the distance recorded by a Huawei Watch 2 Sport is typically half that reported by a Samsung Gear S2 for the same individual and activity session, according to a recent research by a consumer watchdog (72).

Other recent surveys of the accuracy of a range of existing, commercial mobile PA tracking solutions revealed wide variations in reported activity data between different products $(73,74)$. None of the wearables tested in these surveys included, or triangulated, observations (calibrated to the individual's current fitness level) from heart rate sensors, which could have improved the accuracy of these devices. [It should be noted that some of the latest smartwatches, such as the 2019 Asus VivoWatch SP fitness watch (75), incorporate cardiovascular sensors and algorithms of reasonable accuracy (heart rate, ECG, $\mathrm{SpO}_{2}$ and even blood pressure in some smartwatches) that can, when combined with the right software/apps, significantly improve the accuracy of mobile PA tracking.]

Also, automatic exercise type detection/segmentation [e.g., walking (and its intensity: slow, brisk, running, stairs climbing?) vs. cycling (road slope: level ground, up/hill climbing, down?) $v s$. swimming $v s$. playing golf or other types of sports, etc.] remains under-developed in the current generation of trackers [relying here on user's self-reporting/ user's diaries, logs and questionnaires to document exercise type, duration and intensity is equally inaccurate (76)].

As far as absolute sensor/device accuracy is practically concerned, e.g., in tracking the exact actual number of steps walked, one can argue that a very high level of accuracy is not always the highest priority depending on the application and purpose of the tracking, especially when our goal is to monitor user's progress/increased PA and weekly PA duration over time (same person/using same device), rather than absolute figures/steps and calorie counts.

\section{More caveats: lack of a social component, inability to communicate with specialized hardware/sensors and lack of truly individualized feedback}

A recent study (77) included in its summary of the 'state of the art' section a comparative review of smartphone apps for health and fitness and examined each app along the following parameters: App utility and usability for regular training, instructional quality of apps, sensor data usage, and motivation. They also identified some of the most significant drawbacks of the currently available mobile 
applications on the market: many of apps do not have multiuser support or a user community (social component, which has been found to be very important for ensuring sustained PA levels over time without relapse), and only a few applications are compatible with sports hardware, such as heart rate monitors or wearable sensors. Most of the tested health and fitness applications did not use the smartphone's built in sensors, although almost all modern smartphones integrate multiple accelerometers, magnetometers and gyroscopes (and some even have a built-in dedicated pedometer). Device sensor usage could very conveniently enable more functionality, without the need to buy, connect and synchronise (and battery-power) additional hardware incorporating similar sensors.

Another negative point is the fact that many of the current health and fitness apps only partially focus on longterm motivation, so far by using social connections, e.g., to beat a friend's results, collaborate with friends to achieve a common goal (e.g., a total of 100,000 steps a day within a group of 15-20 individuals) or to challenge a stranger (or mostly to promote the app on social media by sharing one's PA achievements on Facebook, Twitter, etc.). Unfortunately, they very rarely go beyond that to offer truly individualized and personalized feedback (see later below), which, if provided, could in fact be a strong factor in the long-term motivation of the user.

\section{Privacy and security issues}

Given the plethora of fitness trackers data, and often realtime syncing IoT (Internet of Things) cloud features, data privacy and military security issues have collided. Recent Strava fitness data from troops wearing their wearable technology, and their associated 'heatmaps', revealed secret military bases and troop movement (78). If these exposed data were available to the public, and given to opposing forces, it could have put military and civilians at risk of being attacked.

Privacy concerns extend beyond sharing user location updates with cloud services. In fact, if we were to remove all location data from a user's profile, there would still be other possible ways to reveal the real identity of that user. In a recent study, Kupin et al. (79) collected data at dozens of time points in virtual reality (VR) environments (think digital exergaming here), and could identify an individual with $90 \%$ accuracy. Just like postcode, IP address and voiceprint, VR and augmented reality tracking data should be treated as potential 'personally identifiable information' since they can be used to uncover a person's identity, either alone or combined with other personal identifying details.

PA planning and tracking apps might also act as a vehicle for serving malware/spyware/spam/ads (behavioural tracking of users). Some of them drain the phone's battery very fast either because of lack of optimisation and/or additional background processing that is not related to the app's advertised purpose, e.g., to serve malware or ads.

\section{Shorter battery life}

Despite the numerous health monitoring and data collection options, the Apple Watch typically will not last 24 hours on a single charge. As Apple continues to optimize processes, hardware, and software, one would assume the charge will last longer. Also new hardware features (released with each model) are not enabled until later in the device's life cycle after a software update, including the newly updated Noise app (39).

\section{Looking into the future: desiderata for the ultimate mobile PA tracking solution}

Our proposed desiderata cover four dimensions:

(I) Accuracy improvements: PA segmentation, sensor triangulation, incorporation of indirect calorimetry (individualized calibration), tracking cardiac fitness and muscle strength/mass, etc.;

(II) Precision and accuracy health (Figure 2): delivering truly individualized PA advice and coaching;

(III) Precision and accuracy public health (Figure 2): providing public health decision makers with community-level PA indicators obtained from population app data aggregates;

(IV) Comprehensively addressing user's privacy concerns: revealing user's location, frequency of visits, travel routes are just some of the datacentric concerns that should be adequately addressed.

\section{Delivering precision and accuracy personal bealth: ingredients that should be incorporated in future apps}

The ideal activity tracker/app and platform should offer more accurate, advanced and battery-powerefficient activity tracking and segmentation (automatic PA type, e.g., walking, running, cycling, swimming, etc., and intensity level detection). This can be achieved by combining/triangulating inertial sensors and GPS with 

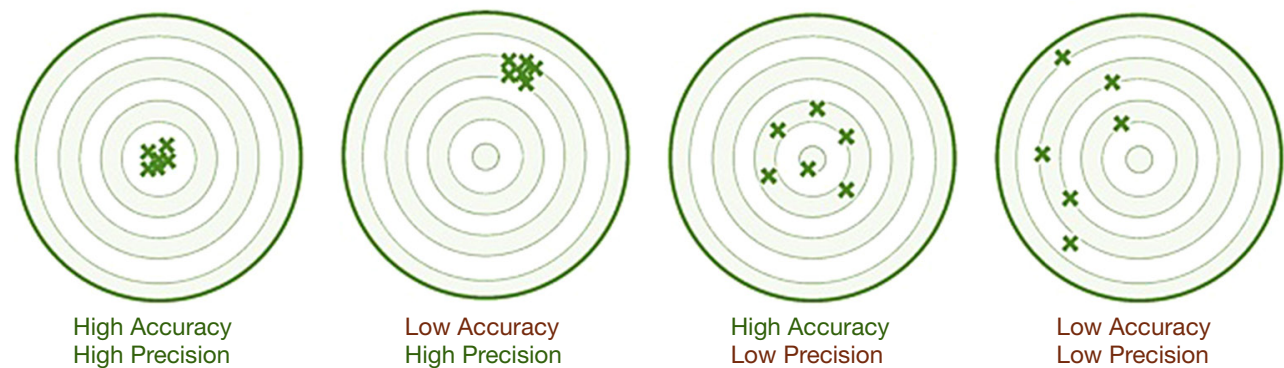

Figure 2 Why the authors prefer the phrase term 'precision and accuracy [medicine/health/public health]' over the more common phrase term 'precision [medicine/health/public health]'. Both precision and accuracy are needed for correct decision making.

heart rate monitoring (also useful for accurately measuring cardiovascular fitness progress) and an optional further individualized calibration (curve) using mobile indirect calorimetry (80) (see later below), building and capitalising on existing smartphone sensor hardware as much as is possible (to reduce the need for extra inertial sensors and extra battery power to run them).

The ultimate solution for activity monitoring that is yet to be developed/released would seamlessly and transparently track the user's PA during the day, while the user is using the phone (flexibly, either in the pocket or on the waist, as the user prefers) and/or wristband/smartwatch, and would automatically differentiate (segment) the periods of walking, running and cycling, as well as the moments when the user was standing, sitting or lying down or in a moving vehicle (sedentary periods) (Figure 3).

For the periods of active ambulation, e.g., walking, running and cycling [or even swimming, using a special waterproof smartphone case and belt (or other form) to fix the phone to the body, or, alternatively a waterproof wireless wearable/wristband or smartwatch], the activity monitor will automatically determine the nature (type and intensity) of the PA being undertaken, and also calculate, where applicable, the number of the steps taken, the distance travelled, and the average speed, as well as the metabolic equivalents (METs) and the corresponding energy expenditure. Accurate exercise segmentation will be made possible by intelligently triangulating real-time multisensor data (GPS/smartphone location services, inertial sensors, heart rate, etc.). In the event the user does not use the phone or wristband while doing some exercise, it would still be possible to manually register a period of exercise by specifying the type of exercise (from a list of possible exercises and workouts) and the duration of the exercise.

Based on the Compendium of Physical Activity (83) (a compilation of several types of exercise and the correspondent METs value) and the user's calibration (see below) and previously known levels (if any) for similar activities, we can then calculate the calories burned. For each period of PA, we can also classify the intensity of PA, using the METs value, into: light ( $<3 \mathrm{METs}$ ), moderate (3-6 METs) and vigorous (>6 METs) (84). For each intensity level, it is possible to calculate the percentages of calories from fat and carbohydrates in the total calories burned. These percentages are dependent not only on the intensity of the activity, but also on the individual's magnitude of effort required to perform a given activity at a given level. This 'magnitude of effort' depends on the individual's previous exercise experience and her/his relative level of fitness (should improve with time), monitored using specialized sensors for that purpose, such as a heart rate monitor.

In computing total energy expenditure over a given period of time, an element of 'individual calibration' will also be needed to more accurately account for a user's baseline energy expenditure during rest and BMR (Basal Metabolic Rate) level. These values vary between and among individuals and even in the same individual, e.g., when they lose weight. Indirect calorimetry is the gold standard for measuring metabolism on an individual (more accurate) basis, by measuring carbon dioxide production and oxygen consumption during rest and activity, but can be cumbersome to implement as a device for routine daily use in natural user's environments/outdoors. However, instead of approximate 'calibration' guess work, the ideal solution should incorporate an option for a mobile indirect calorimetry/metabolism tracker to be used by new users when they first start using the platform (to calibrate and further individualise the energy expenditure computation algorithms for them), followed by intermittent use thereafter as necessary (or as indicated 


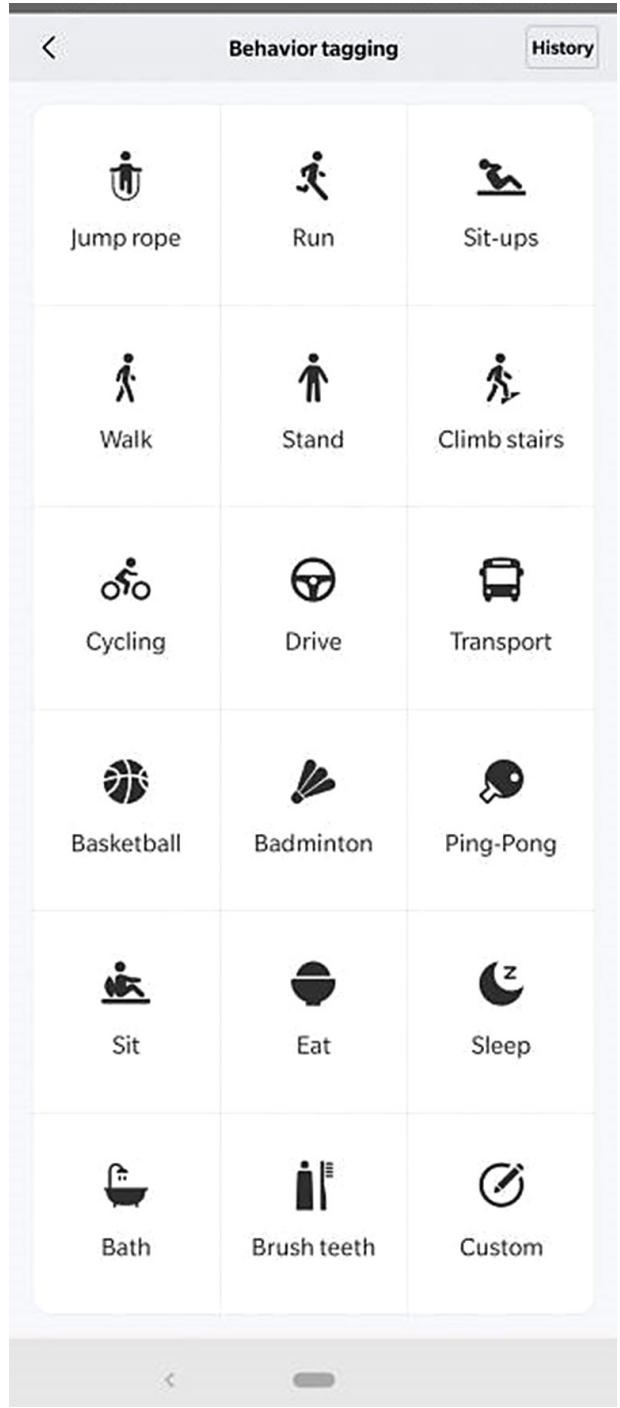

Figure 3 A step towards future automatic segmentation of PA type and intensity: Screenshot of Mi Fit app [Huami/Xiaomi, China, version 4.0.11-November 2019 (81)] is enlisting the help of its users to manually tag different types of daily activities and sports as they are carrying them out, so that the corresponding fitness band sensor data patterns [e.g., from the tri-axial accelerometer, tri-axial gyroscope and PPG (photoplethysmography) heart rate sensors in Xiaomi/Huami Mi Smart Band 4] can be learned by the platform. Moreover, the Xiaomi/Huami Mi Smart Band 4 itself (82) has an on-screen 'Workout' menu where users can select the type of sports activity they are about to start and then tell the band when they have finished their selected sports session (by pressing an on-screen 'Stop' button on the band). Hopefully, future versions of these fitness trackers and their corresponding software platforms will support automatic segmentation of PA types without manual user input. It is noteworthy that Mi Fit is compatible with the Google Fit platform and can upload its user data to the latter over the cloud. by treating clinician), such as when there is a significant change in body weight after several months, i.e., not as a long-term/'carry everyday' wearable. Fully mobile, lowcost solutions such as 'Breezing' (85) are currently close to commercial production.

Muscle mass and strength/torque can act as a good proxy to overall health and fitness status. Skeletal muscle follows the 'use it or lose it principle' and a sedentary lifestyle can give rise to significant muscle loss for those muscles having reduced levels of activity. Lean body mass/muscle strength and torque and percentage of body fat (especially abdominal) should be factored into the computations and individualized recommendations of the ideal PA planning and tracking solution. Regularly feeding progress over time in these departments (muscle mass/strength and body fat) to users can help keep them motivated without relapse. (These aspects will ideally require affordable, user-friendly, noninvasive methods/sensors to be made available for reliably measuring them at the user's own place.)

The ideal app's truly individualized PA plans should also take into consideration the person's age, lifestyle and preferences, neighbourhood characteristics, current PA (baseline) types and levels, individual metabolism (via mobile indirect calorimetry-as mentioned above), genetic profiles, general health status and any relevant existing clinical condition(s) s/he might have, particularly conditions that might affect or be affected by the type and duration of PA to be prescribed to the user (critical ingredients that are largely lacking in today's apps). Indeed, not all calories burned through PA are the same. Recent work by Lin et al. (86) suggests that regular jogging, followed by mountain climbing, walking, power walking, certain types of dancing, and long yoga practices were helpful in maintaining desirable weight ranges in individuals who are genetically predisposed to obesity. But the same study found that cycling, stretching exercises, swimming and 'Dance Dance Revolution' (a digital exergame series) did not counteract the genetic effects on obesity.

Lastly, the prospects of using artificial intelligence (AI) in understanding and acting upon users' PA (big) data are immense. One specialized example is Asensei rowing coach (87), a new (as of 2019) app that is leveraging Olympians and professional athletes' expertise, mobile position sensing, and AI to provide on-demand real-time coaching and feedback. While rowing on a 'Concept2' rowing ergometer, Asensei uses the phone's front face cameras to derive data points to assess movement quality, posture, and stroke rate. To assist the user in rowing towards $\mathrm{AI}$ generated goals, feedback is 
given accordingly, and if doing a live-workout, feedback can be provided by the live coach.

\section{An ecosystem of very many apps benefiting from the same core functionalities}

The ideal app/platform (could also be "baked" into the mobile operating systems) would serve as a 'hub' for a large ecosystem of third-party apps that offer extra features (e.g., different route planning options, different workouts, various exergames to play, etc.) to satisfy the needs of various users-the WeChat platform and the plethora of mini-apps available for it from third-party developers. The ultimate platform would be the one that exposes its core advanced exercise tracking/segmentation and individualized recommender functionalities for other third-party apps to further leverage them and deliver extra value at minimal cost, by just focusing on delivering additional and special features needed by subgroups of users (such as exergames or specialized workouts) without having to 'reinvent the wheel' of exercise segmentation and recommender routines.

\section{Precision and accuracy public bealth: supporting informed public bealth decision-making}

The ideal app/platform should expose a standardized API, with suitable individual privacy provisions/guarantees, data warehousing policies and data sharing arrangements, to enable public health authorities to obtain (geo-tagged) population app data aggregates of PA levels and trends over time amongst their target populations (indicators of community PA level/status).

Population PA indicators can include average steps or, additionally, if Google Fit (42) is used, average 'Move Minutes' and 'Heart Points', per person/per day in different age groups and country regions, cities and city neighbourhoods; percentages of poor, average and high performers in target population; also cumulative (total) population steps, 'Move Minutes' and 'Heart Points' per region, city and neighbourhood for a given period.

Other relevant population-level indicators related to heart rate variability (88), sedentary behaviour and sleep quality/patterns $(89,90)$ in the community can also be extracted from fitness and PA app and gadget big data aggregates of user populations to provide additional 'lifestyle intelligence' for designing better public health interventions.

These indicators can be monitored and compared every few months to determine population progress and trends over time, or at different times of the year/seasons, or following specific PA interventions-population-level surges observed in fitness tracker step counts coinciding with the release of Pokémon Go mobile exergame in July 2016 (Pokémon Go in this example can be considered as a gamified PA "population intervention") $(91,92)$. \{Similarly, sweatcoins [from the aforementioned Sweatcoin app (47)] can serve both as an incentivized PA population intervention (93) and as another community-level PA indicator (population sweatcoin levels as a proxy for population response/ intervention uptake levels and success).\}

Indicators can also be normalized by population number to compare different regions, cities and neighbourhoods. Further interesting patterns might emerge from user data aggregates that can help city planners better understand and optimise the PA landscape of their city and its population; for example, data might reveal that residents of neighbourhoods A and B are doing most of their daily exercises in some walkable streets, parks or public gym facilities in a different neighbourhood C.

Location-tagging of app data aggregates (e.g., by neighbourhood or city) should be straightforward and readily available, as most PA and fitness mobile apps today require 'location permission' and use it to log user's home base location, fetch corresponding weather data, map walking, running and cycling routes, etc. Location is derived from GPS and other location services [Wi-Fi, Bluetooth (beacons) and cellular network] on smartphones, as well as GPS on smart bands/smartwatches that are equipped with one. Moreover, many smart bands/smartwatches without GPS offer a 'connected GPS' mode for tracking location (waypoints), distance and pace using the GPS on the smartphone (app) they are paired to.

In the same vein, Fillekes et al. (94) are trying to develop a comprehensive set of GPS-based indicators reflecting the multidimensional nature of daily mobility for applications in health and aging research.

Ideally, PA tracking in populations and PA mapping of facilities and opportunities in the neighbourhood environment should be segmented by type of activity, e.g., walking, cycling and swimming (86), taking into consideration the different genetic makeups, as well as other relevant profiles and characteristics of population subgroups. If we want to deliver effective and truly individualized PA advice and coaching, and plan existing, and optimize future, community PA facilities and spaces accordingly, we have to include this sort of detail resolution 


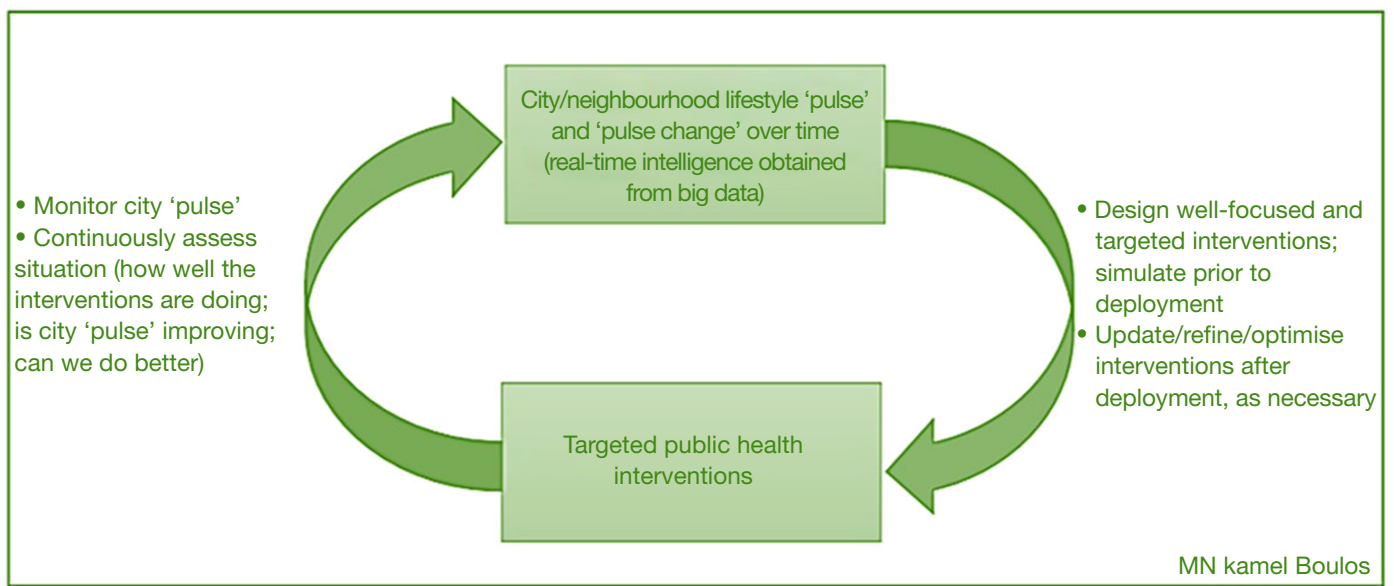

Figure 4 Using big data (location-tagged population data aggregates from PA and fitness apps/gadgets, as well as other relevant data sources and themes, e.g., regularly updated population health records data aggregates [to document baseline levels/needs and track long-term population health improvements], datasets of neighbourhood characteristics/environment, etc.) to support informed public health decisionmaking and evidence-based programme planning, design and evaluation. This approach can result in dynamically optimized programmes that deliver better results with better value for money.

about current opportunities and uptake of different PA types by different population subgroups in the neighbourhood, since not all PA types are equally effective in different types of individuals (86).

Apple's Heart and Movement Study launched in November 2019 is using heart rate/rhythm and movement/ workout big data collected from the Apple Watches and iPhones of 500,000 participants over 5 years to study mobility factors affecting heart health, with the ultimate goal (as stated by Apple) of developing better preventive health tools (95).

Indeed, such big data features and the population insights they provide should help public health authorities and urban planners:

(I) Devise superior PA intervention programmes aimed at both individuals in target populations and at their PA environment/neighbourhood characteristics, e.g., better urban plans to improve neighbourhood walkability, location and versatility optimization of sports/gym facilities and parks according to identified needs, city bike sharing scheme introduction or expansion, etc. (Big data can also help us perform computer simulations of proposed interventions and their effects, to tweak them as necessary prior to real-world deployment and minimise costly failures);

(II) Better target interventions by delivering them to the right audiences/locations in the most effective way, e.g., deliver and prioritize highly tailored PA campaigns to different population subgroups according to their profiles and identified needs (customized PA messages and workout plans in various media formats for different age groups, gamified digital interventions such as (59) to motivate more sedentary population groups, etc.);

(III) And dynamically monitor the effects of deployed programmes over space and time, making adjustments to the interventions as necessary. This cycle is shown in Figure 4.

The choice of app(s)/platform(s) to forge data sharing agreements with, and aggregate/analyse user population data from, will depend on which app(s)/platform(s) are adopted the most by users/by the majority of target populations. Programmes can also be launched to actively promote particular apps/platforms among target populations.

Again, as far as sensor/device accuracy is practically concerned, in population aggregates, we are looking for crude trends over time (e.g., overall population PA levels, and whether they are increasing or decreasing or remaining unchanged over time/in different seasons of the year, etc.), so the inaccuracies and differences between different sensors and device models should not pose a big issue, and most over- and underestimates will offset each other in sufficiently large samples. 


\section{Conclusions}

PA is a critical component in population health. Appropriate physical exercise is essential for good health and disease prevention, but not, (especially when solely relied upon), for weight maintenance or loss. In fact, the "wrong" type of PA might sometimes result in weight gain [e.g., swimming in cold water may stimulate appetite and prevent weight loss (86)]. Tying PA promotion messages to calorie burning for weight loss (as the sole purpose of exercising) can be counterproductive and demotivating for some persons who fail to lose weight with exercise, resulting in these people giving up completely on exercise despite its critical importance for their health [e.g., a systematic review and meta-analysis published in November 2019 found that any amount of running, even just once a week, substantially reduces risk of early death from cardiovascular problems, cancer and other causes and is clearly better than no running (96)].

Leveraging mobile technology, GPS data and creative story- or goal-driven outcomes can be a persuasive way to move more and be entertained simultaneously. Understanding the potential and limitations to mHealth technology and circumstances is crucial to the success of healthcare professionals, game developers, and technologists alike. The ultimate mobile PA planning and tracking app/platform will be the one capable of supporting both precision and accuracy health (offering truly individualized PA advice and coaching while preserving user privacy) and precision and accuracy public health (providing public health decision makers with community-level PA indicators obtained from user population app aggregates).

\section{Acknowledgments}

Funding: None.

\section{Footnote}

Conflicts of Interest: Both authors have completed the ICMJE uniform disclosure form (available at http://dx.doi. org/10.21037/mhealth.2020.01.01). The authors have no conflicts of interest to declare.

Ethical Statement: The authors are accountable for all aspects of the work in ensuring that questions related to the accuracy or integrity of any part of the work are appropriately investigated and resolved.
Open Access Statement: This is an Open Access article distributed in accordance with the Creative Commons Attribution-NonCommercial-NoDerivs 4.0 International License (CC BY-NC-ND 4.0), which permits the noncommercial replication and distribution of the article with the strict proviso that no changes or edits are made and the original work is properly cited (including links to both the formal publication through the relevant DOI and the license). See: https://creativecommons.org/licenses/by-nc-nd/4.0/.

\section{References}

1. Brownell $\mathrm{KD}$, Werner $\mathrm{K}$. The perils of ignoring history: Big tobacco played dirty and millions died. How similar is big food? The Milbank Quarterly. 2009;87:259-94.

2. Malhotra A, Noakes T, Phinney S. It is time to bust the myth of physical inactivity and obesity: you cannot outrun a bad diet. Br J Sports Med 2015;49:967-8.

3. Belluz J, Zarracina J. Why you shouldn't exercise to lose weight, explained with 60+ studies. Vox.

October 31, 2017. Available online: https://www.vox. com/2016/4/28/11518804/weight-loss-exercise-mythburn-calories

4. Basu S, Yoffe P, Hills N. The relationship of sugar to population-level diabetes prevalence: an econometric analysis of repeated cross-sectional data. PLoS One 2013;8:e57873.

5. Kolb H, Martin S. Environmental/lifestyle factors in the pathogenesis and prevention of type 2 diabetes. BMC Med 2017;15:131.

6. Schutte NM, Nederend I, Hudziak J, et al. Heritability of the affective response to exercise and its correlation to exercise behavior. Psychol Sport Exerc 2017;31:139-48.

7. Bauman AE, Reis R, Sallis J, et al. Correlates of physical activity: why are some people physically active and others not? Lancet 2012;380:258-71.

8. Cox D. Watch your step: why the 10,000 daily goal is built on bad science. The Guardian. September 3, 2018. Available online: http://www.theguardian.com/ lifeandstyle/2018/sep/03/watch-your-step-why-the-10000daily-goal-is-built-on-bad-science

9. Public Health England. Guidance - Health matters: getting every adult active every day. July 19, 2016. Available online: https://www.gov.uk/government/ publications/health-matters-getting-every-adult-activeevery-day/health-matters-getting-every-adult-activeevery-day

10. Lee IM, Shiroma E, Kamada M. Association of step 
volume and intensity with all-cause mortality in older women. JAMA Intern Med 2019;179:1105-12.

11. ARION - Transform your running technique (wearable and app). Available online: https://www.arion.run/

12. MapMyRun (app). Available online: https://www. mapmyrun.com/

13. Nike Run Club (iOS app). Available online: https://apps. apple.com/us/app/nike-running/id387771637

14. Runkeeper - Track your runs, walks and more (app). Available online: http://runkeeper.com/

15. Strava - Run and Cycling Tracking on the Social Network for Athletes. Available online: https://www.strava.com/

16. Adidas Runtastic: adidas Running \& Training apps. Available online: https://www.runtastic.com/

17. Sports Tracker (app). Available online: http://www.sportstracker.com/

18. ANT+ in Phones and Tablets. Available online: https:// www.thisisant.com/consumer/ant-101/ant-in-phones

19. Sensoria Home Page (apps, smart garments and platform). Available online: https://www.sensoriafitness.com/

20. RockMyRun (app). Available online: http://www. rockmyrun.com/

21. Fiit: Bring the gym home (app). Available online: https:// fiit.tv/

22. Fitbod (app). Available online: https://www.fitbod.me/

23. Jefit - Gym workout app. Available online: http://www. jefit.com/

24. Johnson \& Johnson Official 7 Minute Workout App. Available online: https://7minuteworkout.jnj.com/

25. Daily Burn - Thousands of Workouts Video. Available online: https://dailyburn.com

26. Peloton ${ }^{\circledR}$ Exercise Bike With Indoor Cycling Classes Streamed Live \& On-Demand. Available online: https:// www.onepeloton.com

27. Wellbeats Virtual Fitness Training. Available online: https://wellbeats.com/

28. Zwift - At Home Cycling \& Running Virtual Training \& Workout Game App. Available online: https://zwift.com/

29. Raphael R. The next Peloton? A look at the players in the digital fitness landscape. April 19, 2019. Available online: https://www.fastcompany.com/90324688/thenext-peloton-a-look-at-the-players-in-the-digital-fitnesslandscape.

30. CycléoONE - Cottos Medical (France). Available online: https://cottos.fr/produit-cycleo/ - https://youtu.be/ ueyNqpPlE4c

31. Cycléo by Cottos - Instagram photos by MNK Boulos. Posted: October 26, 2017. Available online: https://www.
instagram.com/p/BateurWBAXW/

32. Schellewald A. Why do we watch others play? understanding eSports as sporting entertainment. In: Online proceedings of: Seminar on eSports, exergaming, and fantasy leagues, November 2018, University of Jyväskylä, Finland. Available online: https://www.jyu.fi/ hytk/fi/laitokset/mutku/tutkimus/konferenssit-seminaarit/ seminar-on-e-sports-exergaming-and-fantasy-leagues/ programme/esports-seminar-abstracts_18-11.pdf

33. Liao S. Fortnite gives away $\$ 3$ million to its first-ever solo world champion. July 28, 2019. Available online: https:// www.cnn.com/2019/07/28/business/fortnite-solo-winnerbugha-world-cup/index.html

34. GamesRadar (YouTube channel). Fortnite World Cup Solo finals highlights (video). July 28, 2019. Available online: https://www.youtube.com/watch?v=AXfkqXamJU0

35. AIR by Plume Labs - Air Pollution Forecasts On Your Smartphone. Available online: https://plumelabs.com/en/air/

36. BreezoMeter - Accurate Air Quality, Pollen \& Active Fires Information. Available online: https://breezometer.com/

37. Klarify.me (Denmark). Available online: https://uk.klarify.me

38. BreezoMeter - Air Quality API. Available online: https:// breezometer.com/products/air-quality-api

39. Bell K. Apple Watch's new Noise app is unbelievably accurate. June 17, 2019. Available online: https:// www.cultofmac.com/631960/apple-watch-noise-appunbelievably-accurate/

40. Pilates - Android Apps on Google Play (search results). Available online: https://play.google.com/store/ search?q=pilates\&c=apps

41. Sworkit - At Home Workout and Fitness Plans. Available online: https://sworkit.com/

42. Google Fit (app). Available online: https://www.google. $\mathrm{com} / \mathrm{fit} /$

43. Works with Google Fit - Android Apps on Google Play (search results). Available online: https://play.google.com/ store/apps/collection/promotion_3000e6f_googlefit_all

44. Staying Fit with WeRun on WeChat. Official WeChat Blog. May 5, 2017. Available online: https://blog.wechat. com/tag/werun/

45. Duclos M, Oppert J, Verges B, et al. Physical activity and type 2 diabetes. recommendations of the SFD (Francophone Diabetes Society) diabetes and physical activity working group. Diabetes Metab. 2013;39:205-16.

46. Karantonis DM, Narayanan M, Mathie M, et al. Implementation of a real-time human movement classifier using a triaxial accelerometer for ambulatory monitoring. IEEE Trans Inf Technol Biomed 2006;10:156-67. 
47. Sweatcoin - It pays to walk (app). Available online: https:// sweatco.in/

48. Hauk C. Massachusetts woman says Apple Watch saved her life while paddle boarding. June 6, 2019. Available online: https://www.mactrast.com/2019/06/massachusettswoman-says-apple-watch-saved-her-life-while-paddleboarding/

49. Campbell M. Apple Watch credited with saving life days after ECG feature launched in Europe. April 2, 2019. Available online: https://appleinsider.com/ articles/19/04/02/apple-watch-credited-with-saving-lifedays-after-ecg-feature-launched-in-europe

50. Miller C. Apple Watch fall detection helps save epileptic user's life. July 31, 2019. Available online: https://9to5mac. com/2019/07/31/apple-watch-fall-detection-epilepsy/

51. Gonzalez R. The Apple Watch is now the control center for your health. June 4, 2019. Available online: https:// www.wired.com/story/the-apple-watch-is-now-thecontrol-center-for-your-health/

52. Apple ResearchKit and CareKit. Available online: https:// www.apple.com/researchkit/

53. Lineaweaver N. Apple and Aetna announce a new watchdriven wellness program. January 30, 2019. Available online: https://www.businessinsider.com/apple-watchaetna-partnership-2019-1

54. Muoio D. dacadoo, UAE insurer launch activity mobile game, community step challenge. March 5, 2019. Available online: https://www.mobihealthnews.com/content/ dacadoo-uae-insurer-launch-activity-mobile-gamecommunity-step-challenge

55. Boulos MN, Yang S. Exergames for health and fitness: the roles of GPS and geosocial apps. Int J Health Geogr 2013;12:18.

56. Kamel Boulos MN. On the promises, challenges and risks of Pokémon Go and similar geosocial (locationbased) exergames. In: Proceedings of GEOMED 2017, the 10th International Conference on Spatial Statistics, Spatial Epidemiology \& Spatial Aspects of Public Health, September 2017, Porto, Portugal. Available online: https:// www.slideshare.net/sl.medic/on-the-promises-challengesand-risks-of-pokmon-go-and-similar-geosociallocationbased-exergames

57. Wokamon - Monster Walk Quest, The Pedometer Fitness Game (app). Available online: http://www.wokamon.com/

58. Zombies, Run! game (app). Available online: https:// zombiesrungame.com/

59. The Walk - Game by Six to Start and Naomi Alderman (app, created with the NHS and Department of Health).
Available online: https://thewalkgame.com/

60. Iqbal M. Pokémon GO revenue and usage statistics (2019). May 10, 2019. Available online: https://www. businessofapps.com/data/pokemon-go-statistics/

61. Ingress Prime game (app). Available online: https://www. ingress.com

62. Harry Potter Wizards Unite game (app). Available online: https://www.harrypotterwizardsunite.com/

63. Anguita D, Ghio A, Oneto L, et al. Human activity recognition on smartphones using a multiclass hardwarefriendly support vector machine. In: Bravo J, Hervás R, Rodríguez M, et al. editors. Ambient Assisted Living and Home Care. IWAAL 2012. Lecture Notes in Computer Science. Berlin, Heidelberg: Springer, 2012:216-23.

64. Aguiar B, Silva J, Rocha T, et al. Monitoring physical activity and energy expenditure with smartphones. IEEEEMBS International Conference on Biomedical and Health Informatics (BHI), Valencia, 2014:664-7.

65. Fitbit Activity Trackers. Available online: http://www.fitbit. com/

66. Smith C. Nike FuelBand: The rise and fall of the wearable that started it all. February 22, 2016. Available online: https://www.wareable.com/fitness-trackers/not-so-happybirthday-nike-fuelband-2351

67. Omron Activity Monitors. Available online: https://www. omron-healthcare.com/eu/activity-monitors

68. Apple Watch Series 5 (successor of Series 4). Available online: https://www.apple.com/apple-watch-series-5/

69. Bower G. Why sports apparel brands are giving up on fitness apps [Opinion]. Cult of Mac. August 20, 2019. Available online: https://www.cultofmac.com/645646/whysports-apparel-brands-bail-on-fitness-apps/

70. Woods R. Google reportedly abandoned its smartwatch plans years ago. Neowin. September 18, 2019. Available online: https://www.neowin.net/news/google-reportedlyabandoned-its-smartwatch-plans-years-ago

71. Osterloh R. Google to acquire Fitbit. Google Blog. November 1, 2019. Available online: https://blog.google/ products/hardware/agreement-with-fitbit

72. Kleinman Z. Fitness trackers 'add miles to your marathon'. BBC [Internet]. April 27, 2019. Available online: https:// www.bbc.com/news/technology-48072255

73. Case MA, Burwick H, Volpp K, et al. Accuracy of smartphone applications and wearable devices for tracking physical activity data. JAMA 2015;313:625-6.

74. Lewington L. Putting fitness trackers to the test. BBC [Internet]. February 10, 2015. Available online: https:// www.bbc.com/news/technology-31113602 
75. ASUS VivoWatch SP - Instagram post by MNK Boulos. September 6, 2019. Available online: https://www. instagram.com/p/B2DJUnpn16V/

76. Prince SA, Adamo KB, Hamel ME, et al. A comparison of direct versus self-report measures for assessing physical activity in adults: a systematic review. Int J Behav Nutr Phys Act 2008;5:56.

77. Kranz M, Möller A, Hammerla N, et al. The mobile fitness coach: towards individualized skill assessment using personalized mobile devices. Pervasive Mob Comput 2013;9:203-15.

78. Hsu J. The Strava heat map shows even militaries can't keep secrets from social data. Wired (online). January 29, 2018. Available online: https://www.wired.com/story/ strava-heat-map-military-bases-fitness-trackers-privacy/

79. Kupin A, Moeller B, Jiang Y, et al. Task-Driven Biometric Authentication of Users in Virtual Reality (VR) Environments. In: Kompatsiaris I, Huet B, Mezaris V, et al. editors. MultiMedia Modeling. MMM 2019. Lecture Notes in Computer Science. Berlin: Springer, 2019:55-67.

80. Bridgeman D, Xian X, Tsow F, et al. Personalized indirect calorimeter for energy expenditure (EE) measurement. Glob J Obes Diabetes Metab Syndr 2015;2:004-8.

81. Mi Fit (app). Available online: https://play.google.com/ store/apps/details?id=com.xiaomi.hm.health - https://apps. apple.com/us/app/mi-fit/id938688461

82. Mi Smart Band 4. Available online: https://www.mi.com/ global/mi-smart-band-4

83. Ainsworth BE, Haskell W, Herrmann S, et al. 2011 compendium of physical activities: a second update of codes and MET values. Med Sci Sports Exerc 2011;43:1575-81.

84. WHO. What is Moderate-intensity and Vigorous-intensity Physical Activity? Available online: http://www.who.int/ dietphysicalactivity/physical_activity_intensity/en/

85. Breezing - The world's only mobile metabolism tracker. Available online: http://breezing.com/

86. Lin WY, Chan CC, Liu YL, et al. Performing different kinds of physical exercise differentially attenuates the genetic effects on obesity measures: Evidence from 18,424 Taiwan Biobank participants. PLoS Genet 2019; 15:e1008277.

87. Asensei. Available online: https://asensei.com/

88. Campos M. Heart rate variability: A new way to track well-being. Blog post. November 22, 2017; updated
October 22, 2019. Available online: https://www.health. harvard.edu/blog/heart-rate-variability-new-way-trackwell-2017112212789

89. Roberts KC, Butler G, Branchard B, et al. The Physical Activity, Sedentary Behaviour and Sleep (PASS) Indicator Framework. Health Promot Chronic Dis Prev Can 2017;37:252-6.

90. Butler GP, Roberts KC, Kropac E, et al. At-a-glance - Conceptualizing a framework for the surveillance of physical activity, sedentary behaviour and sleep in Canada. Health Promot Chronic Dis Prev Can 2019;39:201-4.

91. Silver K. Pokemon Go leading to a 'population-level' surge in fitness tracker step counts. July 15, 2016. Available online: https://www.washingtonpost.com/news/toyour-health/wp/2016/07/15/pokemon-go-leading-to-apopulation-level-surge-in-fitness-tracker-step-counts/

92. Freeman K. Cardiogram users exercise more with release of Pokémon Go. July 13, 2016. Available online: https:// watchaware.com/post/17592/cardiogram-users-exercisemore-with-release-of-pokemon-go

93. Elliott M, Eck F, Khmelev E, et al. Physical Activity Behavior Change Driven by Engagement With an Incentive-Based App: Evaluating the Impact of Sweatcoin. JMIR Mhealth Uhealth 2019;7:e12445.

94. Fillekes MP, Giannouli E, Kim EK, et al. Towards a comprehensive set of GPS-based indicators reflecting the multidimensional nature of daily mobility for applications in health and aging research. Int J Health Geogr 2019;18:17.

95. Comstock J. Apple rolls out Research: One app for three new longitudinal studies. MobileHealthNews. November 14, 2019. Available online: https://www.mobihealthnews. com/news/north-america/apple-rolls-out-research-oneapp-three-new-longitudinal-studies

96. Pedisic Z, Shrestha N, Kovalchik S, et al. Is running associated with a lower risk of all-cause, cardiovascular and cancer mortality, and is the more the better? A systematic review and meta-analysis. Br J Sports Med 2020;54:898-905.

doi: $10.21037 /$ mhealth.2020.01.01

Cite this article as: Kamel Boulos MN, Yang SP. Mobile physical activity planning and tracking: a brief overview of current options and desiderata for future solutions. mHealth 2021;7:13. 